\title{
HSV-UL18 DNA vaccine construction and immunodetection.
}

\author{
Sinan Fang ${ }^{1-34}$, Qiaoli Wang ${ }^{1,24}$, Lu Wang $^{1-3}$, Fan Luo ${ }^{1-3}$, Junwei Liu ${ }^{1-3}$, Ju Jin $^{1,2}$, Lin Jin ${ }^{4}$, Zhe Ren ${ }^{1,2 *}$, Yifei Wang ${ }^{1,2}$ \\ ${ }^{1}$ Guangzhou Jinan Biomedicine Research and Development Center, National Engineering Research Center of Genetic \\ Medicine, Jinan University, Guangzhou, Guangdong, China \\ ${ }^{2}$ Key Laboratory of Bioengineering Medicine, Guangzhou, Guangdong, China \\ ${ }^{3}$ College of Life Science and Technology, Jinan University, Guangzhou, Guangdong, China \\ ${ }^{4}$ Division of Hematology and Oncology, Department of Medicine, UAB Comprehensive Cancer Center, University of \\ Alabama at Birmingham, Birmingham, AL, USA \\ \#Authors contributed equally to this work
}

\begin{abstract}
Herpes simplex virus (HSV-1) is a common pathogen that causes skin and venereal diseases. To date, a cure for this pathogen does not exist. Therefore, the development of a vaccine to prevent infection with the virus seems to be the best solution. DNA vaccines represent a novel vaccine formulation, which is able to induce an immune response. In the present study, we used genetic engineering technologies to ligate the HSV-1 protein Vp23 into the pcDNA3.1 plasmid, resulting in the constructed plasmid pcDNA3.1-UL18, the potential DNA vaccine against HSV1.The pcDNA3.1-UL18 (p-Vp23) was used for mice immunization. Determination of serum IgG levels, serum neutralization antibody titers, proliferation of spleen lymphocytes, Delayed Hypersensitivity Test reaction, as well as detection of the inflammatory factors IFN-, IL-2, IL-4 and IL-10 in spleen cells revealed that the constructed p-Vp23, to a certain extent, was able to induce immune response in mice.
\end{abstract}

Keywords: DNA vaccine, Herpes simplex virus1, UL-18, Animal immunization.

Accepted on May 08, 2017

\section{Introduction}

Herpes simplex virus (HSV) is a globally spreading DNA virus that can cause oral and genital ulcers [1]. Few cases have shown that HSV infections can lead to complications, including meningitis, encephalitis, neonatal infections, and keratitis [2]. Furthermore, previous studies indicate that HSV patients possess a three times increased risk of an infection with the human immunodeficiency virus (HIV), compared to healthy people [3]. Genital ulcers caused by HSV can be treated with antiviral Acyclovir. However, this can only suppress multiple recurrent ulcers, and is not able to completely treat the HSV infection [4].The virus first infects the skin or the epithelial cells of the mucous membranes and finally enters the nerve cells and in the latent [5]. Therefore, the best strategy to deal with the virus is to prevent and control the infection of HSV.

Atpresent, there are many studies reporting the development of HSV vaccines, however, no substance has been reported so far. Some studies have been carried out in clinical trials [6,7]. For example, the herpevac test has investigated the truncated viral glycoprotein D2 $(\mathrm{gD} 2 \mathrm{t})$ as a potential vaccine in more than $8,000 \mathrm{HSV}-1 / \mathrm{HSV}-2$ seronegative women. The vaccine showed 58\% efficacy in preventing HSV-1 genital infections, as well as $32 \%$ effectiveness in the prevention of HSV-1 infection [8]. Although there have been some achievements, the prevention efficiency is not high. From 1990 Wolff found DNA vaccine so far, based on continuous improvement of genetic engineering techniques, DNA vaccines have developed rapidly, from the first generation of attenuated, inactivated vaccines, and the second generation subunit vaccines, to the current development of recombinant DNA vaccines [9]. DNA vaccines have many advantages, including low cost, high security, and high availability; hence, they have recently gained much attention [10-12]. The VP23 capsid protein, encoded by the HSV UL18 gene, contains 318 amino acids. Two VP23 proteins bind the VP19C protein, forming a ternary complex, which is the main component of the virion nucleocapsid protein structural frame work. A previous study has shown that this complex is particularly important for the proliferation of the HSV-1 virus [13]. Therefore, we decided to use genetic engineering techniques to ligate the HSV-1 specific protein UL18 to the pcDNA3.1 plasmid, resulting in pcDNA3.1-UL18 (p-Vp23), the potential DNA recombinant vaccine of the HSV-1. Furthermore, we investigated the immune effect in mice, aiming to provide a new strategy for the development of a clinical HSV vaccine. 


\section{Materials and Methods}

\section{Viruses, cells and plamids}

The virus strain used for our experiments was herpes simplex virus type 1 (HSV-1 F strain) ATCCVR733. African green monkey kidney cells (Vero) (ATCC CCLS1), purchased from American Type Culture Collection, were maintained in Dulbecco's Modified Eagle Medium (gibco, USA) supplemented with 10\%fetal bovine serum (FBS No. 130908, Zhejiang Tianhang Biological Technology Co., China) at 5\% $\mathrm{CO}_{2}$ in a humidified $37^{\circ} \mathrm{C}$ incubator. The median tissue culture infection dose $\left(\mathrm{TC}_{\mathrm{ID} 50}\right)$ of ATCC VR733 used in the cytopathic assay was $10^{-5.5} / 100 \mu \mathrm{L}$. E. coli stain DH5 $\alpha$, as a cloning host, was used for plasmid transformation and extraction. All strains were grown in Luria Broth (LB). Plasmids pcDNA3.1, BAC, and BAC-HSV-1 are reserved in our laboratory.

\section{Construction of plasmid pcDNA3.1-UL18}

The HSV-1 genome was used as a template to amplify the UL18 fragment by PCR, using primers UL18 F( $5^{\prime}$-ATA GAATTCATGCTGGCGGACGGCTTTGAAACT-3') and UL18 R (5'-TAGGGATCCTTAGGGATAGCGTATAACGGGGGC-3'). Restriction of the UL18 fragment as well as the eukaryotic vector pcDNA3.1 was performed by EcoR1 (TaKaRa Code: D3320) and BamH1 (TaKaRa Code: D3321) restriction enzymes. Using the T4 DNA ligase (TaKaRa Code: D2011A), the restricted fragments were connected to construct the pcDNA3.1-UL18 plasmid. Sequencing by Sangon Biotech (Shanghai, CHINA) Co., Ltd.

\section{Animal immunity}

Bilateral musculus biceps brachii injections were conducted in male Kunming mice (Laboratory Animals Monitoring Institute, Guangdong) at 5 weeks of age, with $100 \mu \mathrm{g}$ per mouse administered three immunizations were performed in 21 days, at intervals of 7 days. There were 4 groups, namely the negative control, positive control, normal control and experimental group, which received BAC, BACHSV-1, PBS (Phosphate Buffered Saline) and pcDNA3.1UL18 respectively. One week post the third triplicatevaccination, blood was collected from the orbits and splenic lymphocytes were extracted in each group.

\section{Collection of animal blood and spleen cells}

One week after the last immunization, all mice were sacrificed. Afterwards, orbital blood was collected from eyeballs, centrifuged at $4^{\circ} \mathrm{C}$ for $5 \mathrm{~min}$ at $4000 \mathrm{rpm}$. The supernatant was used for the neutralizing antibody assays and ELISA serum IgG content experiments. Furthermore, spleens were removed from the mice by aseptically opening the abdominal cavity. To collect the spleen cells, the organs were immersed in DMEM and, after addition of collagenase, were cut into pieces for $30 \mathrm{~min}$ using the 80 mesh screen. After centrifugation at $1000 \mathrm{rpm}$ for $10 \mathrm{~min}$, the supernatant was discarded; the cells were resuspended in DMEM and incubated for $5 \mathrm{~min}$. After washing the cells twice with DMEM, $1 \mathrm{ml}$ of $3.6 \% \mathrm{NaCl}$ was added and the cells were counted to ensure a cell density of $5 \times 10^{6}$ cells $/ \mathrm{mL}$. The treated cells were used for cell factor determination and spleen lymphocyte proliferation assays.

\section{Neutralization test}

The HSV-1 virus was diluted 1:100 and mixed with dilutions of inactivated mouse serum (1:100 to $1: 6400)$ in $2 \%$ serum-free medium. Vero cells were incubated with 100 $\mu l$ of the diluted serum mixed with the virus per well. Each sample was tested three times in duplicates. The virus was used as a positive control, while the pre-immune serum was used as a negative control. The cell culture was incubated for 5 days. Using neutralizing antibody titers calculated by the Reed-Muench method, the 1:100 dilution of the serum should be able to inhibit the CPE (cytopathic effect) and neutralize $50 \%$ of the virus. The vitality to neutralize $50 \%$ of the virus as standard. The highest dilution of serum inhibits $50 \%$ of the virus as antibody titers.

\section{Detection of serum IgG by ELISA}

The inactivated coating liquid was mixedwith $100 \mu$ lwhole HSV-1 antigen and was kept at $4{ }^{\circ} \mathrm{C}$ overnight. $3 \%$ Albumin from bovine serum (BSA) 5 component closed at $25^{\circ} \mathrm{C} 2 \mathrm{~h}$. PBST (PBS add $0.05 \% \mathrm{v} / \mathrm{v}$ Tween 20 ) washed three times. Afterwards, $100 \mu \mathrm{l}$ of different serum dilutions (1:400, 1:800, 1:1600,1:3200,1:6400,1:12800,1:25600) were added. The pre-immune serum (1:100 dilutions) was used as a negative control. After $1 \mathrm{~h}$ incubation at room temperature, the plate was washed with PBST three times. Then $100 \mu \mathrm{l}$ of HRP-goat anti-mouse IgG (1:1000 dilutions) was added and the plate was again incubated at room temperature for $1 \mathrm{~h}$ and washed with PBST three times. Finally, $100 \mu \mathrm{l}$ of ano-phenylendiamine colored solution was added and the reaction was placed in the dark for $10 \mathrm{~min}$. The reaction was terminated using 0.2 $\mathrm{M} \mathrm{H}_{2} \mathrm{SO}_{4}$ and the optical density was measured at $492 \mathrm{~nm}$. Antibody titers were defined as: negative control $\mathrm{OD}_{492}<0.1$, the logarithm of the maximum dilution of serum at the $\mathrm{P} / \mathrm{N} \geq$ 2.1. [P is the OD value of the experimental group, $\mathrm{N}$ for the corresponding negative control group OD value]

\section{Cytokine experiments}

The obtained spleen cell suspension was adjusted to a concentration of $5 \times 10^{5} \mathrm{cells} / \mathrm{mL}$ and was seeded into a 24well cell culture plate, using $1 \mathrm{ml}$ of cell suspension per well. The appropriate sample was added after $24 \mathrm{~h}$, with a final concentration of $10 \mu \mathrm{g} / \mathrm{mL}$. Triple-distilled water was used as a negative control. After incubation for $48 \mathrm{~h}$, the culture supernatant was removed and utilized to measure IFN- $\gamma$, IL2, IL-4 and IL-10 secretion levels using ELISA kits.

\section{Spleen cell proliferation assay}

The cell density of isolated splenocytes was $1 \times 10^{5}$ and the cells were then inoculated into a 96-well cell culture plate, using $100 \mu \mathrm{l}$ per well. After $24 \mathrm{~h}$ of incubation, 100 $\mu \mathrm{l}$ of the corresponding sample solution was added to the experimental group, with a final concentration of $10 \mu \mathrm{g} / \mathrm{mL}$. Triple-distilled water was used as a negative control, while the untreated cells were used as the control group. After $72 \mathrm{~h}$, the MTT (3-(4,5)-dimethylthiahiazo (-z-y1)-3,5-di- 
phenytetrazoliumromide) proliferation assay was performed to calculate the stimulation index (SI):

$\mathrm{SI}=(\mathrm{OD}$ value of the experimental group) / (OD value of the negative control)

\section{Delayed hypersensitivity test (DTH)}

One week after the last immunization, $10 \mu \mathrm{l}$ of inactivated HSV-1 virus suspension was injected into the right ear of each mouse. After centrifugation, the same volume of the supernatant of a cell lysate was injected into the left ear. After $48 \mathrm{~h}$, ear thickness was measured using a caliper. Data are represented as DTH, which is calculated by subtracting the measurement for the left subcutaneous auricle from the right one.

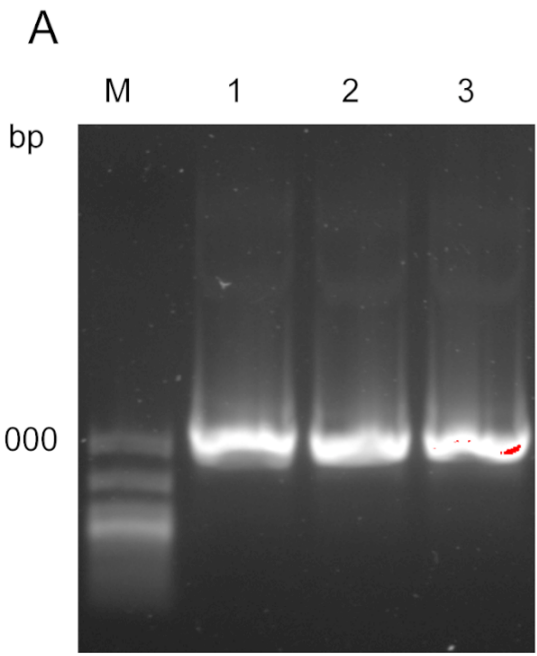

\section{Results}

\section{Construction of plasmid pcDNA3.1-UL18}

Amplification of the UL18 gene from the HSV-1 F strain ATCC VR733 revealed the expected 957-bp PCR product (Figure 1A). The restriction digest of the constructed plasmid pcDNA3.1UL18 showed the expected single bands of the original plasmid pcDNA 3.1 and the inserted UL18 gene. Furthermore, correct ligation of the plasmid was confirmed by sequencing. The sequencing results are shown in supplementary materials (Figure S1).

\section{Blood immunoassay}

The results of the neutralization test and the IgG ELISA are shown in Figures $2 \mathrm{~A}$ and $2 \mathrm{~B}$, respectively. Even with

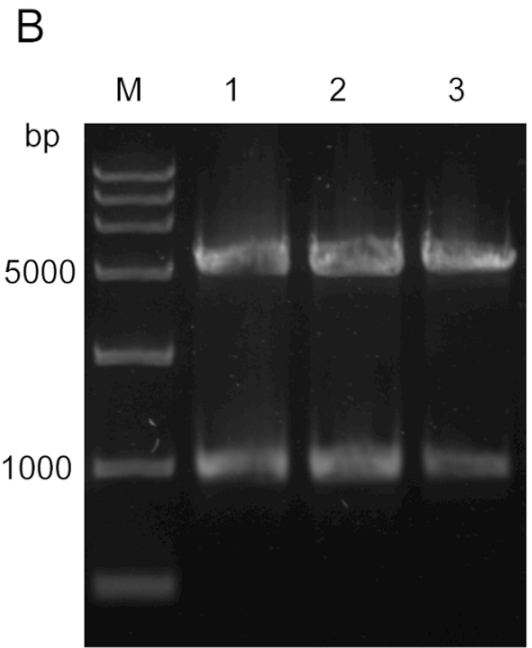

Figure 1. Construction of plasmid pcDNA3.1-UL18.

(A) Lane M: 1000 bp DNA marker; lanes 1, 2, 3: PCR product of the UL18 gene (957 bp).

(B) Lane M: 15000 bp DNA marker; lanes 1, 2, 3: Restriction analysis of pcDNA3.1-UL18.

A

\begin{tabular}{l|llll}
\hline & $p-V p 23$ & $\begin{array}{l}\text { BAC- } \\
\text { HSV-1 }\end{array}$ & BAC & $\begin{array}{l}\text { No- } \\
\text { Treated }\end{array}$ \\
\hline 100 & ++++ & ++++ & ++++ & ++++ \\
200 & ++++ & ++++ & ++++ & ++++ \\
400 & ++++ & ++++ & ++++ & ++++ \\
800 & ++++ & ++++ & ++++ & ++++ \\
1600 & ++++ & ++++ & ++++ & ++++ \\
3200 & ++++ & ++++ & ++++ & ++++ \\
6400 & ++++ & ++++ & ++++ & ++++ \\
HSV-1 & ++++ & ++++ & ++++ & ++++ \\
Cell & -- & - & -- & -- \\
\hline
\end{tabular}

B

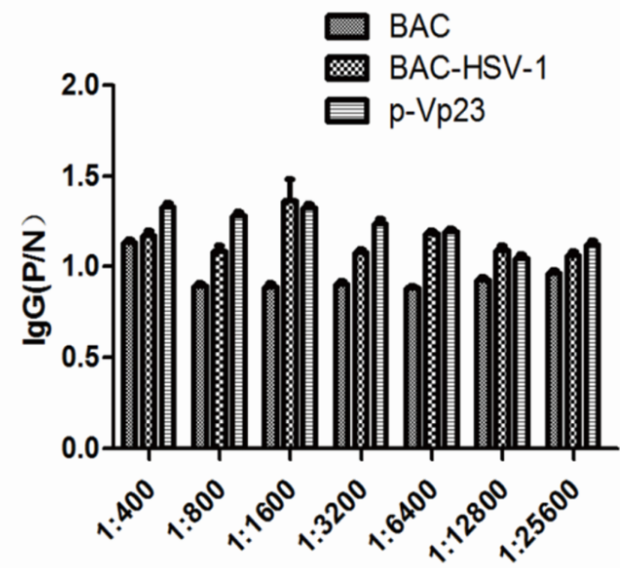

Figure 2. Mice blood neutralizing antibody and $\operatorname{IgG}$ levels.

(A) Neutralizing antibody test. Investigated dilutions of inactivated mouse serum: 1:100 to 1:6400. The HSV-1 virus suspension was mixed with the dilutions of the serum, which was then used to inoculatethe Vero cells. Highest dilution of serum inhibits the CPE as antibody titers. The vitality to neutralize 50\% of the virus as standard, with reed-muench calculated neutralizing antibody titers. “++++" represents viral infection. “--" represents no virus infection.

(B) ELISA for detection of serum IgG. IgG levels of the experimental group (p-Vp23), the positive control group (BAC-HSV-1), and the negative control group (BAC) are shown. The results revealed no significant changes in IgG antibody levels. 
the lowest serum dilution (1:100), the experimental group p-Vp23 and the positive control group BAC-HSV-1 were unable to suppress $50 \%$ of the HSV-1 lesions (Figure $2 \mathrm{~A}$ ), indicating that there were no antibodies present in the serum after immunization. Furthermore, the ELISA assay revealed no significant changes in the serum IgG levels in p-Vp23 compared to BAC-HSV-1 and BAC, confirming a lack of HSV-1 antibody production in the mice after immunization.

\section{Spleen cell immunity}

To further analyze the p-Vp23 immune effect, we investigated the proliferation of spleen cells and the production of four inflammatory cytokines. The proliferation assay revealed significant differences between the negative control group BAC, compared to the experimental group p-Vp23 $(* * \mathrm{p}<0.01)$ and the positive control group BACHSV-1 $(* \mathrm{p}<0.05)$, respectively (Figure $3 \mathrm{~A})$. Furthermore, the inflammatory factors assay observed significant changes in IFN- $\gamma(* * p<0.01)$, as well as IL-2 and IL-4 $(* p<0.05)$ production, comparing the positive control group BACHSV-1 with the negative control group (Figure 3B). The experimental group p-Vp23 also showed a significant difference compared to the negative control group, but only with regards to IL-2 production $(* p<0.05)$. There were no significant changes observed in IL-10 production between the groups.

\section{Delayed hypersensitivity test (DTH)}

DTH reaction is an important immune response in the body. It plays a role in removing the exogenous microorganisms.

A

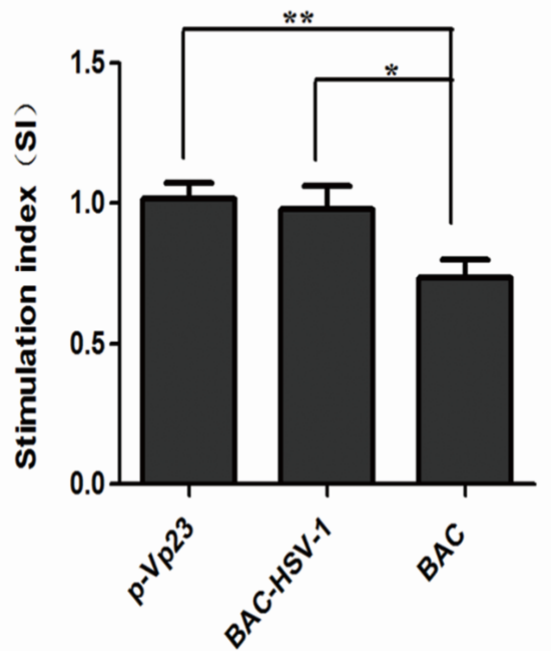

In the present study, we measured ear swelling to reflect the immunity in mice. DTH results show that the experimental group (p-VP23) and the positive control group (BACHSV-1) showed varying degrees of changes in ear thickness, compared to the negative control group (BAC) (Figure 4). Statistical analysis $(n=4)$ showed no significant differences, indicating that the experimental mice exhibited no obvious DTH reaction after immunization.

\section{Discussion}

Since from HSV was discovered, people have been continually seeking the effective treatment regimen of it. The vaccine has been used as the most effective means to prevent viral infection, which need a long process to be developed. This process includes inactivated virus vaccines, live attenuated vaccines, subunit vaccines and peptide vaccines and so on. Even through the technology was improved continually, and all kinds of vaccines have its advantage and disadvantage obviously, it is difficult to find a particularly effective vaccine to apply in clinical. $[1,14]$. The reason may be related to HSV-specific infection mechanism, especially that HSV has a strong latency, making the vaccine cannot be a good prevention or interference with the virus latent. Besides, there is another important reason that attenuated or inactivated virus vaccines cannot fully guarantee the safety itself. As the result of it, scientists are looking for a kind of safe and effective vaccine which was different from traditional vaccines [15]. As the latest vaccine, DNA vaccine has provided a new direction for people to seek HSV vaccine.

DNA vaccine, which has been concerned with the third

B

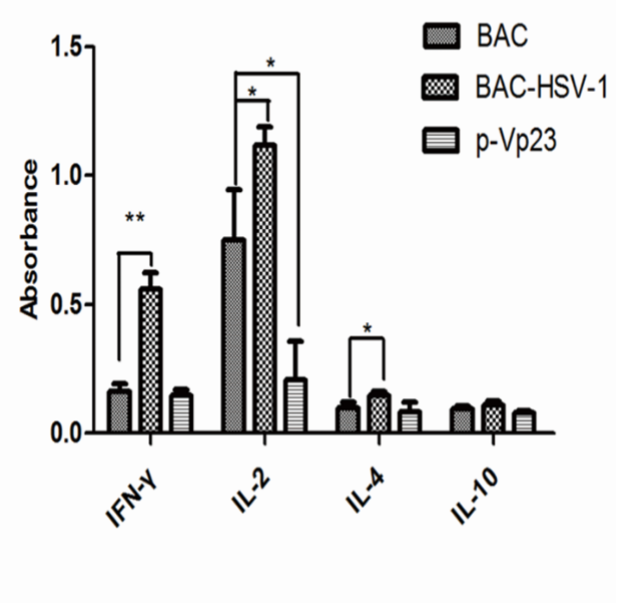

Figure 3. Spleen cell proliferation assay and inflammatory factors assay.

(A) Assay to calculate the cell proliferation stimulation index (SI). It was found that the spleen cell proliferation of the positive control group (BAC-HSV-1) exhibited a significant difference $\left({ }^{*} p<0.05\right)$ compared to that in the negative control group (BAC). Spleen cells of p-Vp23 immunized mice revealed a highly significant difference $\left({ }^{* *} p<0.01\right)$ compared to that in the negative control group (BAC).

(B) Expression of IFN- $\gamma, I L-2, I L-4$ and IL-10 in spleen cells. The p-Vp23 experimental group showed decreased IL-2 expression, compared with the negative control group $(B A C)(* * p<0.01)$, while no significant difference was found for the other cytokines. The positive control group (BAC-HSV-1) showed significant increases in the expression of IFN- $\gamma, I L-2$ and IL-4, compared with the control group (BAC). 


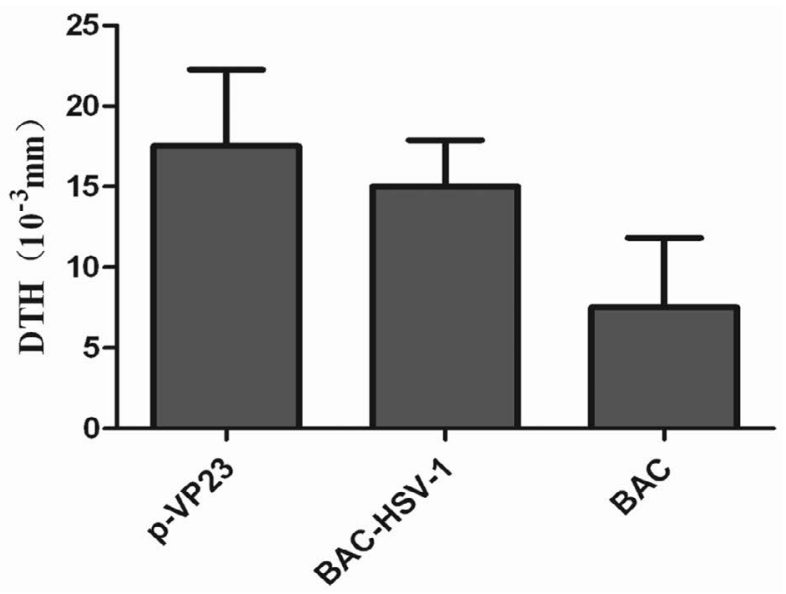

Figure 4. Delayed hypersensitivity test (DTH).

DTH results revealed varying degrees of changes in ear thickness in mice in the experimental group ( $p$-VP23), as well as in the positive control group (BAC-HSV-1), compared to those in the negative control group (BAC). Statistics $(n=4)$ showed

generation of the vaccine since from the development of the traditional vaccine and genetically engineered subunit vaccine, its development get much attention. Its cellular immunity and humoral immunity has been confirmed by related experiments. Such vaccines have some advantage such as its simple preparation process, stability, low toxicity, easy to fold and so on [2]. For example, most of the HSV2 DNA vaccines use HSV-2 two major antigen genes called gD2 and gB2. In 2008, a kind of vaccine called HSV-2gD2 DNA vaccine was subjected to phase I clinical trial, including a double-blind, vector-controlled, dose-increasing with a dose gradient of 100, 300, 1000 and $3000 \mu \mathrm{g}$. The result is no dose-limited toxicity, and only one subject of the highest dose group has been detected a gD2-specific T-cell immune response, indicating that a higher dose was required for the vaccine to produce an immune response [16]. Several recently report of DNA vaccines, such as gD2 plasmid DNA, the DNA of gD2 and DNA of gD2 and gB2CTL, which encode UL46 and UL47, can induce the production of humoral and cellular immune responses successfully and have a strong protective effect on virus attack [17-19].

HSV-1 encodes about 90 proteins, most faction of which was clear. About half of them are necessary for viral replication, others are unnecessary for deletion. Besides, removing the unnecessary genes and substitution of exogenous genes does not affect viral replication, which is the way of traditional virus vaccine to consider. For VP23, one of the four major proteins that make up the viral capsid is transcribed by the essential gene UL18 by a viral replication. It is usually not be targeted as a vaccine. The DNA vaccine for vp23 as a target is not studied. Therefore, we decided to study the UL18 gene, the VP23 capsid protein of HSV-1, to generate a constructed DNA vaccine (pcDNA 3.1-UL18 or p-Vp23) using the eukaryotic expression vector pcDNA3.1.The effect of the vaccine was analyzed in mice by investigation of serum IgG, serum neutralizing antibody titers, lymphocyte proliferation, DTH reaction and ELISA-based detection of the inflammatory cytokines IFN- $\gamma$, IL-2, IL-4 and IL-10.
The neutralization test and the detection of IgG serum levels showed that after $\mathrm{p}-\mathrm{Vp} 23$ immunization, the mice were unable to produce antibodies in the blood. Furthermore, the potential vaccine did not stimulate inflammation in the spleen cells of mice. However, $\mathrm{p}-\mathrm{Vp} 23$ activated the proliferation of the spleen lymphocytes. Taken together, these results showed that $\mathrm{p}-\mathrm{Vp} 23$ can stimulate a part of the immune response in mice, but is not able to activate antibody production in the blood. The development of DNA vaccines has been a major concern, but the specific immune system has not been studied well. A DNA antibody is different from conventional antibodies, as it enters the body via a process of passive absorption instead of an active invasion process [20]. To date, researchers believe that the injected DNA vaccine is degraded in the body and is partly absorbed by the epithelial cells and the muscle cells. In addition, there is a small part of DNA vaccines that circulates in body fluids, which is absorbed by antigen-presenting cells (APC). DNA vaccines are basically constructed from a eukaryotic expression vector, wherein they are absorbed by the cells expressing the corresponding viral proteins. Immune cells recognize the appropriate immune response to produce immune memory [21-23]. There are different factors that might influence the effects of DNA vaccine immunization: (a) the ability of the cells to absorb the DNA vaccine; (b) the protein expression ability of the DNA vaccine virus in the host cell; (c) the expression of the viral protein that can effectively activate the body's immune response $[10,20,24]$. We hypothesize that the possible reason for this effect is that $\mathrm{p}-\mathrm{Vp} 23$ is excessively degraded in mice, owing to which only small amounts of p-Vp23 circulate in body fluids and reach immune tissues. Another factor worth considering regarding in-depth studies of DNA vaccines is the security. For example, foreign DNA can be integrated into the host cell, which might cause mutations that can influence cell proliferation. Furthermore, high amounts of foreign DNA will lead to the formation of anti-DNA antibodies. Although this is unlikely, it cannot be excluded [25,26]. However, the hypersensitivity experiment performed in our study 
confirmed that $\mathrm{p}-\mathrm{Vp} 23$ does not cause allergic reactions and immune system disorders in mice. Furthermore, $\mathrm{p}-\mathrm{Vp} 23$ also did not cause the death of immunized mice, which suggests that $\mathrm{p}-\mathrm{Vp} 23$ is safe as a potential $\mathrm{HSV}-1$ vaccine.

\section{Conclusion}

In our study, we constructed $\mathrm{p}-\mathrm{Vp} 23$, a DNA vaccine against HSV-1, using genetic engineering techniques, which provides a new strategy for potential use in clinical HSV vaccine research.

\section{Acknowledgement}

This work was supported by the National Natural Science Foundation of China [grant numbers 81274170, 81573471]; the Collaborative Innovation Program of Guangdong province of China [grant number2016A040402033] and the Biomedical Research and Development Center of Jinan University.

\section{References}

1. Gupta R, Warren T, Wald A. Genital herpes. Lancet. 2007;370(9605):2127-37.

2. Birkmann A, Zimmermann H. HSV antivirals - Current and future treatment options. Curr Opin Virol. 2016;18:913.

3. Johnston C, Gottliebe SL, Walda A. Status of vaccine research and development of vaccines for herpes simplex virus. Vaccine. 2016;34(26):2948-52.

4. Stanberry LR, Cunningham AL, Mindel A, et al. Prospects for control of herpes simplex virus disease through immunization. J Clin Infect Dis. 2000;30(3):549-66.

5. Tang VA, Rosenthal KL. Intravaginal infection with herpes simplex virus type-2 (HSV-2) generates a functional effector memory $\mathrm{T}$ cell population that persists in the murine genital tract. J Reprod Immunol. 2010;87(12):39-44.

6. Gudmundsdotter L, Wahren B, Haller BK, et al. Amplified antigen-specific immune responses in HIV-linfected individuals in a double blind DNA immunization and therapy interruption trial. Vaccine. 2011;29(33):5558-66.

7. Oxman MN, Levin MJ, Johnson GR, et al. A vaccine to prevent herpes zoster and post herpetic neuralgia in older adults. N Engl J Med. 2005;352(22):2271-84.

8. Belshe RB, Leone PA, Bernstein DI, et al. Efficacy results of a trial of a herpes simplex vaccine. N Engl J Med. 2012;366(1):34-43.

9. Liu MA. Vaccines an historical perspective and view to the future. J Immunol Rev. 2011;239(1):62-84.

10. Dutton JL, Li B, Woo WP, et al. A novel DNA vaccine technology conveying protection against a lethal herpes simplex viral challenge in mice. PLoS ONE. 2013;8(10):e76407.

11. Fowler VL, Barnett PV. Progress in the development of
DNA vaccines against foot-and-mouth disease. J Expert Rev Vaccines. 2012;11(4):481-93.

12. Ferraro B, Morrow MP, Hutnick NA, et al. Clinical applications of DNA vaccines: Current progress. J Clin Infect Dis. 2011;53(3):296-302.

13. Jin F, Li S, Zheng K, et al. Silencing herpes simplex virus type 1 capsid protein encoding genes by siRNA: A promising antiviral therapeutic approach. PLoS ONE. 2014;9(5):e96623.

14. Saade F, Petrovsky N. Technologies for enhanced efficacy of DNA vaccines. J Expert Rev Vaccines. 2012;11(2):189209.

15. Lal H, Cunningham AL, Godeaux O, et al. Efficacy of an adjuvant herpes zoster subunit vaccine in older adults. $\mathrm{N}$ Engl J Med. 2015;372(22):2087-96

16. Cattamanchi A, Posavad CM, Wald A, et al. Phase I study of a herpes simplex virus type 2 (HSV-2)DNA vaccine administered to healthy, HSV-2-seronegative adults by a needle-free injection system. J Clin Vaccine Immunol. 2008;5(11):1638-43.

17. Huilan Y, Cui Z, Jianyong F, et al. Construction of ,and T-helper (Th)1/Th2 immune responses to herpes simplex virus type 2 glycoprotein D-cytotoxic T-lymphocyte epitope DNA vaccine. J Clin Exp Dermatol. 2010;35(5):537-42.

18. Shlapobersky M, Marshak JO, Dong L, et al. Vaxfectinadjuvanted plasmid DNA vaccine improves protection and immunogenicity in a murine model of genital herpes infection. J Gen Virol. 2012;93(Pt6):1305-15.

19. Veselenak RL, Shlapobersky M, Pyles RB, et al. A Vaxfectinadjuvanted HSV-2 plasmid DNA vaccine is effective for prophylactic and therapeutic use in the guinea pig model of genital herpes. Vaccine. 2012;30(49):7046-51.

20. Sardesai NY, Weiner DB. Electroporation delivery of DNA vaccines prospects for success. J Curr Opin Immunol. 2011;23:421-29.

21. Gothelf A, Gehl J. What you always needed to know about electroporation based DNA vaccines. J Hum Vaccin Immunother. 2012;8(11):1694-702.

22. Ishii KJ, Kawagoe T, Koyama S, et al, Tank-binding kinase-1 delineates innate and adaptive immune responses to DNA vaccines. Nature. 2008;451(7179):725-9.

23. Ishikawa H, Ma Z, Barber GN. STING regulates intracellular DNA-mediated, type I interferon-dependent innate immunity. Nature. 2009;461(7265):788-92.

24. Sasaki S, Amara RR, Oran AE, et al, Apoptosis-mediated enhancement of DNA-raised immune responses by mutant caspases. Nat Biotechnol. 2001;19:543-7.

25. Ishii KJ, Weiss WR, Ichino M, et al. Activity and safety of DNA plasmids encoding IL-4 and IFN gamma. J Gene Ther. 1999;6(2):237-44. 
26. Osorio Y, Cohen J, Ghiasi H, et al. Improved protection from primary ocular HSV 1infection and establishment of latency using multigenic DNA vaccines. J Invest Ophthalm Visual Sci. 2004;45(2):506-14.

\section{*Correspondence to:}

Zhe Ren

Guangzhou Jinan Biomedicine Research and Development Center

National Engineering Research Center of Genetic Medicine

Jinan University

Guangzhou, Guangdong

China

Tel: 011-86-02085227715

E-mail: rz62@163.com 